\title{
La edad paterna como factor de riesgo para malformaciones congénitas
}

\author{
Julio $\mathbf{N}$ azer $\mathbf{H}^{1}$, Lucía Cifuentes $\mathbf{0}^{2}$, Francisca Millán $\mathrm{Z}^{\mathrm{a}}$, \\ Paola Vacarisas Aa, Stephanie Köbrich Scha, \\ Alfredo Aguila $\mathbf{R}^{\mathbf{1}}$.
}

\section{Paternal age as a risk factor for congenital malformations}

Background: The role of advanced maternal age as a risk factor for congenital malformations in offspring is known. However, the influence of paternal age is not clear. Aim: To evaluate the association between advanced paternal age and the risk for congenital malformations. Patients and Methods: Analysis of maternal and paternal age of cases (malformed newborns) and controls from ECLAMC Database (Latin American Collaborative Study of Congenital Malformations) registered at the University of Chile Clinical Hospital during the decade from Jan 11997 to Dec 31 2006. Newborns and stillborns were grouped according to maternal age into 6 intervals. In each interval, paternal ages of cases and controls were compared. The inverse procedure was performed to assess maternal age effect. Other variables as gestational age and birth weight were analyzed for the intervals of maternal and paternal ages. Results: No significant differences were observed in paternal age between cases and controls in any of the intervals of maternal age. However, mean maternal age was higher for cases than for controls ( $p=0,0149$ ). Gestational age and birth weight depend more on being case or control than on the age of parents. Conclusions: No differences in paternal age were observed between cases and controls in this series of newborns (Rev Méd Chile 2008; 136: 201-8).

(Key words: Fathers; Infant, newborn; Malformations, congenital)

Recibido el 22 de marzo, 2007. Aceptado el 13 de agosto, 2007.

${ }^{1}$ Unidad de Neonatología, Hospital Clínico Universidad de Chile. Santiago de Chile. ${ }^{2}$ Instituto de Ciencias Biomédicas, Facultad de Medicina, Universidad de Chile.

anterna de Medicina, Facultad de Medicina, Universidad de Chile

$\mathrm{E}^{\mathrm{s}}$ conocida la influencia que sobre la prevalencia de defectos congénitos tienen las edades maternas extremas, tanto adolescentes como edades avanzadas ${ }^{1-6}$. Se ha visto la tendencia en los

Correspondencia a: Dr. Julio Nazer H. Unidad de Neonatología, Hospital Clínico Universidad de Chile. Av. Santos Dumont 999. Santiago, Chile. Fono: 2774106.

E mail: jnazer@redclinicauchile.cl países industrializados de tener hijos a edades más avanzadas. Chile no ha quedado fuera de este fenómeno sociodemográfico. Según el Ministerio de Salud de Chile $^{7}$ y el Instituto Nacional de Estadística (INE) ${ }^{8}$, entre 1997 y 2003 ha habido un cambio en la distribución de las edades maternas y paternas. En efecto, en 1997, el grupo etario de mujeres entre 15 y 29 años aportaban 66,07\% de los recién nacidos vivos (RNV), mientras que en 2003 bajó a 61,76\%. Los hombres del mismo 
grupo etario en 1997 eran los padres de $55,3 \%$ de los RNV, mientras que en 2003 sólo de 49,8\%. El grupo de mujeres de más de 39 años tuvieron 2,58\% de los RNV en 1997 y 3,60\% en 2003. Los hombres de este grupo etario en 1997 aportaron $8,98 \%$ de los RNV y $11,3 \%$ en 2003. Igualmente, Bray $^{9}$ en Inglaterra comunica que en 1993 25\% de los nacimientos tenían padres de edad entre $35 \mathrm{y}$ 54 años, diez años después este valor asciende a $40 \%$. En resumen, en las últimas décadas aumenta la proporción de padres y madres de más de 40 años y disminuyen los menores de 30 años. En publicaciones anteriores ${ }^{3,4}$ hemos comunicado este incremento de los promedios de las edades de las madres de la maternidad del Hospital Clínico de la Universidad de Chile (HCUCH), de 24,8 años en 1973, hasta sobre 29 años en la actualidad.

No se ha podido demostrar que la edad paterna avanzada constituya un factor de riesgo para tener hijos afectados de anomalías cromosómicas, tanto numéricas como estructurales ${ }^{10}$, excepto para trisomía 21, en que se ha comunicado cierta asociación ${ }^{11}$. Se ha relacionado la edad paterna con la calidad del semen, fertilidad disminuida y abortos espontáneos ${ }^{12}$. Teóricamente, el número de divisiones celulares mitóticas (premeióticas) durante la espermatogénesis y su marcado aumento con la edad, comparado con la oogénesis, sería un argumento a favor del riesgo de mutaciones en los gametos de hombres de mayor edad, demostrándose que el aumento del daño del ADN en el espermatozoide de hombres entre 36 y 57 años es tres veces mayor que los hombres menores de 35 años ${ }^{13}$. Ya en 1949, Haldane $^{14}$ expuso esta disparidad en las tasas de mutación entre gametos femeninos y masculinos en la hemofilia. Desde entonces, el efecto de la edad paterna ha sido confirmada para varias enfermedades mendelianas como acondroplasia y síndrome de Apert9,14,15. En varias enfermedades dominantes que afectan a tres genes en particular (receptor de factor de crecimiento de fibroblastos 2 y 3 y el proto-oncogén RET), el riesgo que el hijo se vea afectado aumenta a medida que se incrementa la edad del padre ${ }^{12}$. Otras enfermedades de etiología compleja, como esquizofrenia, también se han asociado al aumento de la edad paterna ${ }^{9,16}$. Sin embargo, la repercusión clínica de estos hechos es menor, reconociéndose efecto real sólo en patologías de baja incidencia, por lo que el riesgo absoluto no es relevante ${ }^{13}$. Dado que el número de nacimientos de parejas de edades avanzadas son numéricamente pocos, el poder estadístico para poder establecer una asociación entre enfermedades genéticas y edad paterna avanzada es limitado ${ }^{17}$. Presentamos aquí nuestra casuística del último decenio.

\section{Objetivos}

Evaluar si existe alguna asociación entre incremento de edad paterna y tasas de prevalencia al nacimiento de malformaciones congénitas en recién nacidos de la maternidad del HCUCH. No la encontramos en nuestra casuística publicada en 19944, pero dado el aumento de las edades paternas en los últimos años, es posible que actualmente podamos detectar esta asociación.

\section{PACIENTES Y MÉTODO}

El Hospital Clínico de la Universidad de Chile es integrante del ECLAMC (Estudio Colaborativo Latino Americano de Malformaciones Congénitas) desde 1969. Se ingresa al registro a todos los recién nacidos (RN) de 500 o más gramos de peso de nacimiento, vivos y mortinatos, que nacen en la maternidad y que presenten una o más malformaciones. Se toma como control al RN del mismo sexo que nace a continuación y que no presente defectos congénitos. Se completa una ficha ad hoc según el Manual Operacional ${ }^{18}$ con sus datos demográficos. Esta ficha se graba en una base de datos y se envía electrónicamente a Río de Janeiro, donde está la Coordinación Central. Se analizará el material de la década enero de 1997 hasta diciembre de 2006.

Existe una correlación positiva entre edad materna y edad paterna, es decir, lo habitual es que a mayor edad de la madre haya también un aumento de la edad del padre. Esto hace difícil establecer cuál de las dos representa el riesgo para el nacimiento de un hijo con malformaciones congénitas. Para intentar resolver esta interrogante, se siguió el siguiente procedimiento estadístico: en primer lugar, para evaluar la importancia de la edad paterna, se agruparon los recién nacidos 
afectados y controles de acuerdo con la edad de la madre, en seis grupos etarios. En cada intervalo etario materno se comparó el promedio de la edad paterna de niños malformados y sus controles respectivos mediante prueba $t$ de Student. A continuación se hizo el procedimiento inverso para evaluar la edad de la madre: se separaron los niños malformados y sus controles según la edad del padre en los mismos seis intervalos etarios. En cada intervalo de edad paterna se comparó la edad promedio de las madres de niños malformados y controles con prueba $t$ de Student. Lo mismo se hizo para comparar promedios de edad gestacional y peso de nacimiento entre casos y controles, en cada uno de los intervalos de edad paterno y materno. Adicionalmente, se hizo un análisis de varianza a tres factores (diagnóstico, edad materna y edad paterna en categorías) para la edad gestacional y el peso de nacimiento.

\section{RESULTADOS}

En el período 1997-2006 ocurrieron 20.123 nacimientos consecutivos en la maternidad del Hospital Clínico de la Universidad de Chile, entre los que se encontró 1.722 recién nacidos $(\mathrm{RN})$ con una 0 más malformaciones congénitas, lo que significa una prevalencia al nacimiento de 8,6\%. El promedio de edad materna del período fue 28,9 años y el promedio de edad paterna 31,3 años.

Del total de nacimientos, $10,33 \%$ fueron pequeños para la edad gestacional (PEG), 13,2\% entre los RN malformados (228/1.722) y $10,1 \%$ entre los controles (140/1.376).

No se encontró diferencias significativas entre las edades paternas promedio de los casos $(31,7$ años) y controles (31,4 años), ni en la muestra total ni en ninguno de los intervalos etarios maternos por separado, incluso en algunos de éstos la edad paterna promedio fue mayor en controles que en casos (Tabla 1).

Los promedios de edades maternas fueron significativamente superiores en casos ( 29,3 años) que en controles ( 28,7 años) ( $p=0,0149)$; al separar la muestra por intervalos etarios paternos, se observa que siempre fue superior el promedio de edad materna en casos que controles, pero este hecho alcanza la significación estadística sólo cuando los padres tenían entre 20 y 24 años (Tabla 2). Esta diferencia de edad materna promedio entre casos y controles fue mucho menor a la que encontrábamos en décadas anteriores en este mismo hospital, cuando las madres atendidas en la maternidad eran más jóvenes que actualmente ${ }^{4-6}$.

El peso de nacimiento fue significativamente inferior en casos $(3.198,02 \mathrm{~g})$ que controles (3.346 g) ( $p<0,0001)$; esta diferencia se manifiesta en casi todos los intervalos de edades paternas y maternas (Tablas 3 y 4). Al analizar el peso de nacimiento promedio en los distintos intervalos de edades maternos, se observa que éste fue aumentando a mayor edad materna hasta los 34 años, después el peso de nacimiento promedio decreció, tanto en casos como en controles. En cambio, este comportamiento no se aprecia con el

Tabla 1. Promedios de edad paterna en recién nacidos malformados y controles según la edad de la madre

\begin{tabular}{|lrrrrrrrr|}
\hline $\begin{array}{l}\text { Intervalo } \\
\text { etario } \\
\text { materno }\end{array}$ & $\mathrm{n}$ & $\begin{array}{c}\text { Casos } \\
\text { prom }\end{array}$ & $\mathrm{DE}$ & $\mathrm{n}$ & $\begin{array}{c}\text { Edad paterna } \\
\text { Controles } \\
\text { prom }\end{array}$ & $\mathrm{DE}$ & $\mathrm{t}$ & $\begin{array}{r}\mathrm{p} \text {-value } \\
\alpha=5 \%\end{array}$ \\
\hline$<20$ & 87 & 21,333 & 4,437 & 116 & 21,569 & 4,268 & $-0,3827$ & 0,7023 \\
$20-24$ & 314 & 25,783 & 4,098 & 305 & 26,256 & 3,979 & $-1,4541$ & 0,1464 \\
$25-29$ & 491 & 30,026 & 4,589 & 502 & 29,723 & 4,641 & 1,0356 & 0,3006 \\
$30-34$ & 440 & 33,948 & 5,039 & 423 & 33,842 & 5,003 & 0,3104 & 0,7564 \\
$35-39$ & 259 & 37,772 & 5,373 & 231 & 38,177 & 4,988 & $-0,8620$ & 0,3891 \\
$\geq 40$ & 101 & 40,347 & 6,418 & 84 & 42,226 & 7,048 & $-1,8968$ & 0,0594 \\
Total & 1.692 & 31,613 & 6,922 & 1.661 & 31,374 & 7,019 & 0,9953 & 0,3197 \\
\hline
\end{tabular}

prom: promedio DE: desviación estándar 
Tabla 2. Promedios de edad materna en recién nacidos malformados y controles, según la edad del padre

\begin{tabular}{|c|c|c|c|c|c|c|c|c|}
\hline \multirow{2}{*}{$\begin{array}{l}\text { Intervalo } \\
\text { etario } \\
\text { paterno }\end{array}$} & \multicolumn{3}{|c|}{ Casos } & \multicolumn{3}{|c|}{$\begin{array}{l}\text { Edad materna } \\
\text { Controles }\end{array}$} & \multirow[t]{2}{*}{$\mathrm{t}$} & \multirow{2}{*}{$\begin{array}{l}p \text {-value } \\
\alpha=5 \%\end{array}$} \\
\hline & $\mathrm{n}$ & prom & $\mathrm{DE}$ & $\mathrm{n}$ & prom & $\mathrm{DE}$ & & \\
\hline$<20$ & 43 & 17,791 & 2,122 & 42 & 17,405 & 1,875 & 0,8878 & 0,3772 \\
\hline $20-24$ & 217 & 22,659 & 4,048 & 212 & 21,892 & 3,620 & 2,0685 & 0,0392 \\
\hline $25-29$ & 414 & 26,256 & 3,727 & 458 & 25,969 & 3,512 & 1,1688 & 0,2428 \\
\hline $30-34$ & 471 & 29,964 & 4,113 & 435 & 29,568 & 4,128 & 1,4457 & 0,1486 \\
\hline $35-39$ & 319 & 33,213 & 4,995 & 316 & 32,991 & 4,446 & 0,5931 & 0,5533 \\
\hline$\geq 40$ & 228 & 36,193 & 4,510 & 198 & 36,308 & 4,683 & $-0,2581$ & 0,7965 \\
\hline Total & 1.692 & 29,262 & 6,200 & 1.661 & 28,743 & 6,145 & 2,4365 & 0,0149 \\
\hline
\end{tabular}

prom: promedio DE: desviación estándar

Tabla 3. Promedio de peso de nacimiento en recién nacidos malformados y controles según la edad del padre

\begin{tabular}{|lrrrrrrrr|}
\hline $\begin{array}{l}\text { Intervalo } \\
\text { etario } \\
\text { paterno }\end{array}$ & $\mathrm{n}$ & $\begin{array}{c}\text { Casos } \\
\text { prom }\end{array}$ & $\mathrm{DE}$ & $\mathrm{n}$ & $\begin{array}{c}\text { Peso al nacer } \\
\text { prom }\end{array}$ & $\mathrm{DE}$ & $\mathrm{t}$ & $\begin{array}{r}\mathrm{p} \text {-value } \\
\alpha=5 \%\end{array}$ \\
\hline$<20$ & 43 & 3173,95 & 765,19 & 42 & 3177,38 & 443,84 & $-0,0252$ & 0,9800 \\
$20-24$ & 216 & 3189,00 & 756,27 & 212 & 3340,09 & 533,34 & $-2,3844$ & 0,0175 \\
$25-29$ & 414 & 3272,29 & 684,32 & 457 & 3356,31 & 544,25 & $-2,0142$ & 0,0443 \\
$30-34$ & 471 & 3155,98 & 749,11 & 433 & 3309,63 & 632,30 & $-3,3177$ & 0,0009 \\
$35-39$ & 319 & 3193,40 & 783,52 & 314 & 3407,17 & 565,70 & $-3,9302$ & $<0,0001$ \\
$\geq 40$ & 227 & 3180,69 & 677,36 & 198 & 3354,85 & 634,23 & $-2,7234$ & 0,0067 \\
Total & 1.690 & 3199,53 & 732,75 & 1.656 & 3346,96 & 580,81 & $-6,4414$ & $<0,0001$ \\
\hline
\end{tabular}

prom: promedio DE: desviación estándar

Tabla 4. Promedio de peso de nacimiento en recién nacidos malformados y controles según la edad de la madre

\begin{tabular}{|lrrrrrrrr|}
\hline $\begin{array}{l}\text { Intervalo } \\
\text { etario } \\
\text { materno }\end{array}$ & $\mathrm{n}$ & $\begin{array}{c}\text { Casos } \\
\text { prom }\end{array}$ & $\mathrm{DE}$ & $\mathrm{n}$ & $\begin{array}{c}\text { Controles } \\
\text { prom }\end{array}$ & $\mathrm{DE}$ & $\mathrm{t}$ & $\begin{array}{r}\mathrm{p} \text {-value } \\
\alpha=5 \%\end{array}$ \\
\hline$<20$ & 89 & 3148,54 & 753,16 & 117 & 3260,98 & 464,67 & $-1,3188$ & 0,1887 \\
$20-24$ & 314 & 3217,83 & 773,18 & 306 & 3321,35 & 541,26 & $-1,9267$ & 0,0545 \\
$25-29$ & 494 & 3224,03 & 720,88 & 501 & 3349,46 & 556,45 & $-3,0746$ & 0,0022 \\
$30-34$ & 441 & 3227,90 & 688,02 & 421 & 3399,86 & 577,59 & $-3,9649$ & $<0,0001$ \\
$35-39$ & 260 & 3158,10 & 745,07 & 231 & 3341,26 & 671,82 & $-2,8468$ & 0,0046 \\
$\geq 40$ & 101 & 3025,07 & 828,45 & 84 & 3288,93 & 718,85 & $-2,2889$ & 0,0232 \\
Total & 1.699 & 3198,02 & 735,45 & 1.660 & 3346,62 & 580,25 & $-6,4915$ & $<0,0001$ \\
\hline
\end{tabular}

prom: promedio

DE: desviación estándar 
aumento de edad paterna. Para evaluar cuál de las variables estudiadas tiene influencia en el peso de nacimiento, se hizo un análisis de varianza a tres factores (condición de caso o control, edad materna y edad paterna); para este efecto se agruparon las edades maternas y paternas de acuerdo a los 6 intervalos descritos en las Tablas 1 y 2 . El análisis de varianza demostró que todas las variables en conjunto inciden en el peso de nacimiento (Tabla 5) y que en forma aislada sólo tendría alguna importancia la condición de sano o control ( $p$ $<0,001$ ), no así las edades maternas y paternas, aunque la edad materna está cerca de la significancia estadística ( $\mathrm{p}=0,071$ ).

La edad gestacional fue significativamente menor en casos que controles ( $p<0,0001$ ), tanto al analizar la muestra completa o al subdividirla según la edad de la madre (Tabla 6), esta diferencia fue especialmente relevante cuando la madre tenía más de 40 años $(0,8$ semanas de diferencia promedio entre casos y controles). Asimismo, la edad gestacional fue menor en casos que controles, en todos los intervalos de edad paterna (Tabla 7), esta diferencia alcanza su mayor magnitud en los padres menores de 20 años (más de una semana de diferencia promedio entre casos y controles) y cuando el padre tenía entre 35 y 39 años. Al analizar el promedio de edad gestacional de acuerdo a la edad materna, se observa que en los casos ésta creció a medida que aumentaba la edad de la madre hasta los 34 años, después de esta edad decrecía; en los controles no

Tabla 5. Análisis de varianza a tres factores (diagnóstico [malformado o control], edad materna y edad paterna) para el peso de nacimiento

\begin{tabular}{|lcrrrr|}
\hline $\begin{array}{l}\text { Fuente de } \\
\text { variación }\end{array}$ & $\begin{array}{c}\text { Grados de } \\
\text { libertad }\end{array}$ & $\begin{array}{c}\text { Suma de } \\
\text { cuadrados }\end{array}$ & $\begin{array}{c}\text { Cuadrados } \\
\text { medios }\end{array}$ & \multicolumn{1}{c|}{ F } & p-value \\
\hline Modelo & 11 & $3,6 \times 10^{10}$ & $3,028 \times 10^{9}$ & 3634,1 & $>0,001$ \\
Diagnóstico & 1 & 13719693,5 & 13719693,5 & 16,5 & $<0,001$ \\
Edad materna & 5 & 8466043,0 & 1693208,6 & 2,0 & 0,071 \\
Edad paterna & 5 & 2152775,7 & 4300555,2 & 0,5 & 0,764 \\
Error & 3.361 & $2,8 \times 10^{9}$ & 833248,9 & & \\
Total & 3.372 & $3,9 \times 10^{10}$ & & & \\
\hline
\end{tabular}

Tabla 6. Edad gestacional promedio de recién nacidos malformados y controles según la edad de la madre

\begin{tabular}{|c|c|c|c|c|c|c|c|c|}
\hline \multirow{2}{*}{$\begin{array}{l}\text { Intervalo } \\
\text { etario } \\
\text { materno }\end{array}$} & \multicolumn{3}{|c|}{ Casos } & \multicolumn{3}{|c|}{$\begin{array}{c}\text { Edad gestacional } \\
\text { Controles }\end{array}$} & \multirow[t]{2}{*}{$\mathrm{t}$} & \multirow{2}{*}{$\begin{array}{r}p \text {-value } \\
\alpha=5 \%\end{array}$} \\
\hline & $\mathrm{n}$ & prom & $\mathrm{DE}$ & $\mathrm{n}$ & prom & $\mathrm{DE}$ & & \\
\hline$<20$ & 57 & 37,842 & 3,217 & 69 & 38,449 & 1,623 & $-1,3714$ & 0,1727 \\
\hline $20-24$ & 189 & 37,921 & 2,953 & 199 & 38,402 & 1,875 & $-1,9267$ & 0,0548 \\
\hline $25-29$ & 341 & 37,974 & 2,641 & 337 & 38,347 & 2,178 & $-2,0082$ & 0,0450 \\
\hline $30-34$ & 300 & 38,030 & 2,427 & 296 & 38,291 & 1,960 & $-1,4409$ & 0,1502 \\
\hline $35-39$ & 175 & 37,617 & 2,561 & 173 & 38,052 & 2,336 & $-1,6544$ & 0,0990 \\
\hline$\geq 40$ & 75 & 37,240 & 3,149 & 66 & 38,136 & 1,847 & $-2,0257$ & 0,0447 \\
\hline Total & 1.137 & 37,870 & 2,700 & 1.140 & 38,291 & 2,048 & $-4,1965$ & $<0,0001$ \\
\hline
\end{tabular}

prom: promedio

DE: desviación estándar 
Tabla 7. Edad gestacional promedio de recién nacidos malformados y controles según la edad del padre

\begin{tabular}{|lrrrrrrrr|}
\hline $\begin{array}{l}\text { Intervalo } \\
\text { etario } \\
\text { paterno }\end{array}$ & $\mathrm{n}$ & $\begin{array}{c}\text { Casos } \\
\text { prom }\end{array}$ & $\mathrm{DE}$ & $\mathrm{n}$ & $\begin{array}{c}\text { Controles } \\
\text { prom }\end{array}$ & $\mathrm{DE}$ & $\mathrm{t}$ & $\begin{array}{l}\mathrm{p} \text {-value } \\
\alpha=5 \%\end{array}$ \\
\hline$<20$ & 32 & 37,313 & 3,788 & 25 & 38,560 & 1,325 & $-1,5705$ & 0,1220 \\
$20-24$ & 132 & 37,970 & 2,556 & 145 & 38,372 & 1,863 & $-1,5077$ & 0,1328 \\
$25-29$ & 266 & 38,143 & 2,580 & 303 & 38,386 & 2,070 & $-1,2467$ & 0,2130 \\
$30-34$ & 327 & 37,786 & 2,955 & 287 & 38,101 & 2,269 & $-1,4665$ & 0,1430 \\
$35-39$ & 221 & 37,620 & 2,757 & 228 & 38,412 & 1,855 & $-3,5830$ & 0,0004 \\
$\geq 40$ & 152 & 37,954 & 2,040 & 150 & 38,153 & 2,117 & $-0,8335$ & 0,4052 \\
Total & 1.130 & 37,868 & 2,705 & 1.138 & 38,291 & 2,050 & $-4,1960$ & $<0,0001$ \\
\hline
\end{tabular}

prom: promedio DE: desviación estándar

se aprecia este incremento pero sí un menor promedio de edad gestacional cuando la madre tiene más de 35 años. El análisis de varianza para la edad gestacional, aparece en la Tabla 8 y demuestra que el único factor relevante por sí solo es la condición de sano o malformado ( $p$ $<0,0001)$. No fue importante la edad de los progenitores.

\section{Discusión}

En el total de la muestra sólo hay diferencia en los promedios de edad materna (Tabla 2), no en la paterna. Sin embargo, esta diferencia de edad materna entre casos y controles es mucho menor a la encontrada entre 1969 y 19794-6 y menor también a la que encontramos entre casos de atresia de esófago ${ }^{19}$, de sexo ambiguo $^{20}$, de malformaciones anorrectales ${ }^{21}$ y de hipospadias ${ }^{22}$ con sus respectivos controles, todos con análisis anteriores a 1997. El promedio de edad materna en las dos décadas anteriores a 1997 oscilaba alrededor de 25 y 27 años, mientras que en la actualidad este promedio está cercano a los 30 años.

Un hecho importante observado es que los promedios de peso de nacimiento, tanto en malformados como en controles, aumenta junto con la edad materna hasta los 35 años, para disminuir progresivamente después. Los pesos de nacimiento son menores en los hijos de madres de edades maternas extremas (menores de $20 \mathrm{y}$ mayores de 40 años).

Un estudio poblacional danés ${ }^{23}$ con casi un millón y medio de nacimientos vivos, concluye

Tabla 8. Análisis de varianza a tres factores (diagnóstico [malformado o control],
edad materna y edad paterna) para la edad gestacional

\begin{tabular}{|lcrrrr|}
\hline $\begin{array}{l}\text { Fuente de } \\
\text { variación }\end{array}$ & $\begin{array}{c}\text { Grados de } \\
\text { libertad }\end{array}$ & $\begin{array}{c}\text { Suma de } \\
\text { cuadrados }\end{array}$ & $\begin{array}{c}\text { Cuadrados } \\
\text { medios }\end{array}$ & F & p-value \\
\hline Modelo & 11 & 3328028,3 & 277335,7 & 47505,8 & $<0,0001$ \\
Diagnóstico & 1 & 105,2 & 105,2 & 18,0 & $<0,0001$ \\
Edad materna & 5 & 52,5 & 10,5 & 1,8 & 0,1100 \\
Edad paterna & 5 & 39,1 & 7,8 & 1,3 & 0,2440 \\
Error & 2.285 & 13339,7 & 5,8 & & \\
Total & 2.296 & 3341368,0 & & & \\
\hline
\end{tabular}


que la edad paterna está asociada con labio leporino y paladar hendido, independientemente de la edad materna. Se sugiere que el mecanismo sería la mutación de un solo gen.

La edad paterna tiene un fuerte efecto en los defectos espermáticos ${ }^{12,16}$. A pesar de estos hallazgos, la evidencia de estudios poblacionales que estudian la asociación entre edad paterna y malformaciones congénitas son escasos y sus resultados no son consistentes. McIntosh ${ }^{24}$ describe un patrón general de aumento de riesgo para defectos del tubo neural, cataratas congénitas, acortamiento de extremidad superior y síndrome de Down. $\mathrm{Zhu}^{25}$ no encontró asociación entre la edad paterna y la prevalencia global de malformaciones congénitas, pero sí observó una asociación significativa entre la edad paterna y malformaciones de las extremidades y síndromes malformativos que afectan múltiples sistemas. Kazaura ${ }^{26}$ no muestra evidencia consistente de que el aumento de la edad paterna sea factor de riesgo para malformaciones congénitas en la descendencia.

En resumen, lo que se conoce actualmente sobre el tema, es que el promedio de edad paterna ha ido aumentando progresivamente en otros países y en Chile y que la implicancia de este hecho sobre la salud pública no ha sido profundamente estudiada ni debatida, principalmente por la limitada evidencia epidemiológica ${ }^{27}$. La acumulación de mutaciones durante la maduración de las células espermáticas en el hombre podrían ser las responsables del creciente riesgo de ciertas enfermedades relacionadas con la edad

\section{REFERENCIAS}

1. Pardo RA, Nazer J, Cifuentes L Prevalencia al nacimiento de malformaciones congénitas y de menor peso de nacimiento en hijos de madres adolescentes. Rev Méd Chile 2003; 131: 1165-72.

2. Nazer J, ANtolin M, JuÁrez ME, Hübner ME, Cifuentes L, CASTLL S, Pardo RA. Prevalencia al nacimiento de aberraciones cromosómicas en el Hospital Clínico de la Universidad de Chile: Período 1990-2001. Rev Méd Chile 2003; 131: 651-8.

3. Nazer J, EAglin MA, Cifuentes L. Incidencia del síndrome de Down en la maternidad del Hospital Clínico de la U. de Chile. Un Registro de 25 años. Rev Méd Chile 1998; 126: 383-90. paterna avanzada. Cada día hay más publicaciones referidas a los efectos de ésta sobre sus hijos, riesgos que incluyen reducción de la fertilidad y aumento del riesgo para ciertos defectos congénitos, como también esquizofrenia y cáncer. Aun así, la evidencia existente a la fecha no demuestra una asociación entre la incidencia global de malformaciones congénitas y el aumento de la edad paterna y tampoco la encontramos en nuestra casuística, aunque esta asociación se haya descrito para algunas patologías en particular.

En conclusión, no demostramos ningún efecto relevante de la edad paterna sobre la incidencia global de RN malformados.

En la casuística revisada en este trabajo se confirman los hallazgos ya descritos en la literatura médica, de que los recién nacidos malformados tienen menor promedio de edad gestacional, de peso de nacimiento y mayor promedio de edad materna que en los controles.

El efecto de la edad de los progenitores sobre el promedio de edad gestacional no demostró ser importante.

La postergación de la maternidad ha determinado que la diferencia de la edad materna entre malformados y controles sea menor que la encontrada en décadas anteriores y que la frecuencia de malformaciones congénitas haya ido en aumento a medida que aumentan los promedios de edad materna ${ }^{4-6}$.

Se confirma que la edad materna ideal para tener hijos con menor riesgo de MFC, y con mejor peso de nacimiento, está entre los 20 y 35 años.

4. Nazer J, Cifuentes L, Ruiz G, Pizarro MT. Edad materna como factor de riesgo para malformaciones congénitas. Rev Méd Chile 1994; 122: 299-303.

5. Nazer J, Cifuentes L, Ruiz G. ¿Están aumentando las malformaciones congénitas? Estudio comparativo de dos períodos: 1971-1977 y 1982-1991. Rev Méd Chile 1993; 121: 1068-74.

6. Nazer J, Hubner ME, Cifuentes L, Ramírez R, Catalán J. Aumento de la incidencia del síndrome de Down y su posible relación con el incremento de la edad materna. Rev Méd Chile 1991; 119: 465-71.

7. Anuario Demográfico 1999. Instituto Nacional de Estadísticas y Ministerio de Salud. Santiago de Chile.

8. Anuario de estadísticas vitales 2003. Instituto Nacional de Estadística. Santiago de Chile 2005. 
9. Bray I, Gunnell D, Davey Smith D. Advanced paternal age: How old is too old? J Epidemiol Community Health 2006; 60: 851-3.

10. Buwe A, Guttenbach M, Schmidt M. Effect of parental age on the frecuency of citogenetic abnormalities in human spermatozoa. Cytogenet Genome Res 2005; 111: 213-28.

11. Jyothy A, Kumar KS, Maшkarhuna GN. Parental age and the origin of extra chromosome 21 in Down syndrome. J Hum Genet 2001; 46: 347-50.

12. Wyrobek AJ, Eskenazi B, Young S, Arnheim N, Tiemann-Boege I, Jabs EW et al. Advancing age has differential effects on DNA damage, chromatin integrity, gene mutations, and aneuploidies in sperm. Proc Natl Acad Sci USA 2006; 103: 9601-6.

13. KüHNERT B, NIESCHLAg E. Reproductive functions of the ageing male. Hum Reprod Update 2004; 10: 327-39.

14. HaLdane JB. The association of characters as a result of inbreeding and linkage. Ann Eugen 1949; 15: 15-23.

15. Risch N, Reich EW, Wishnick MM, McCarthy JG. Spontaneous mutation and parental age in humans. Am J Hum Genet 1987; 41: 218-48.

16. CROW JF. The origins, patterns and implications of human spontaneous mutation. Nat Rev Genet 2000; 1: 40-7.

17. Jung A, Schuppe HC, SchiL WH. Are children of older fathers at risk of genetic disorders? Andrologia 2003; 35: 191-9
18. ECLAMC. Manual Operacional. Ed FioCruz. Río de Janeiro. 2005

19. HÜBnER ME, NAZER J, Cifuentes L Atresia de esófago y malformaciones asociadas. Rev Méd Chile 1999; 127: 655-9.

20. Hübner ME, Nazer J, Cifuentes L. Sexo ambiguo. Rev Méd Chile 2001; 129: 509-14.

21. Nazer J, Hübner ME, Valenzuela P, Cifuentes L Malformaciones anorrectales y sus asociaciones preferentes. Experiencia del Hospital Clínico Universidad de Chile. Período 1979-1999. Rev Méd Chile 2000; 128: 519-25.

22. Nazer J, Cifuentes L, Hübner ME, Ramírez R, Ruiz G, PizarRo MT et al. Estudio epidemiológico de factores asociados a Hipospadias. Rev Méd Chile 1992; 120: 244-249.

23. Biwe C, Skytthe A, VAch W. Parent's age and the risk of oral clefts. Epidemiology 2005; 16: 311-16.

24. McIntosh GC, Olshan AF, Baird PA. Paternal age and the risk of birth defects in offspring. Epidemiology 1995; 6: 282-88.

25. Zhu JL, Madsen KM, Vestergaard M, Olesan AV, OLSEN J. Paternal age and congenital malformations. Hum Reprod 2005; 20: 3173-7.

26. Kazaura M, Lie RT, SkJaerven R. Paternal age and the risk of birth defects in Norway. Ann Epidemiol 2004; 14: 566-70.

27. Cedergren MI, Selbing AJ, KäLÉn BA. Risk factors for cardiovascular malformation: A study based on prospectively collected data. Scand J Work Environ Health 2002; 28: 12-7. 